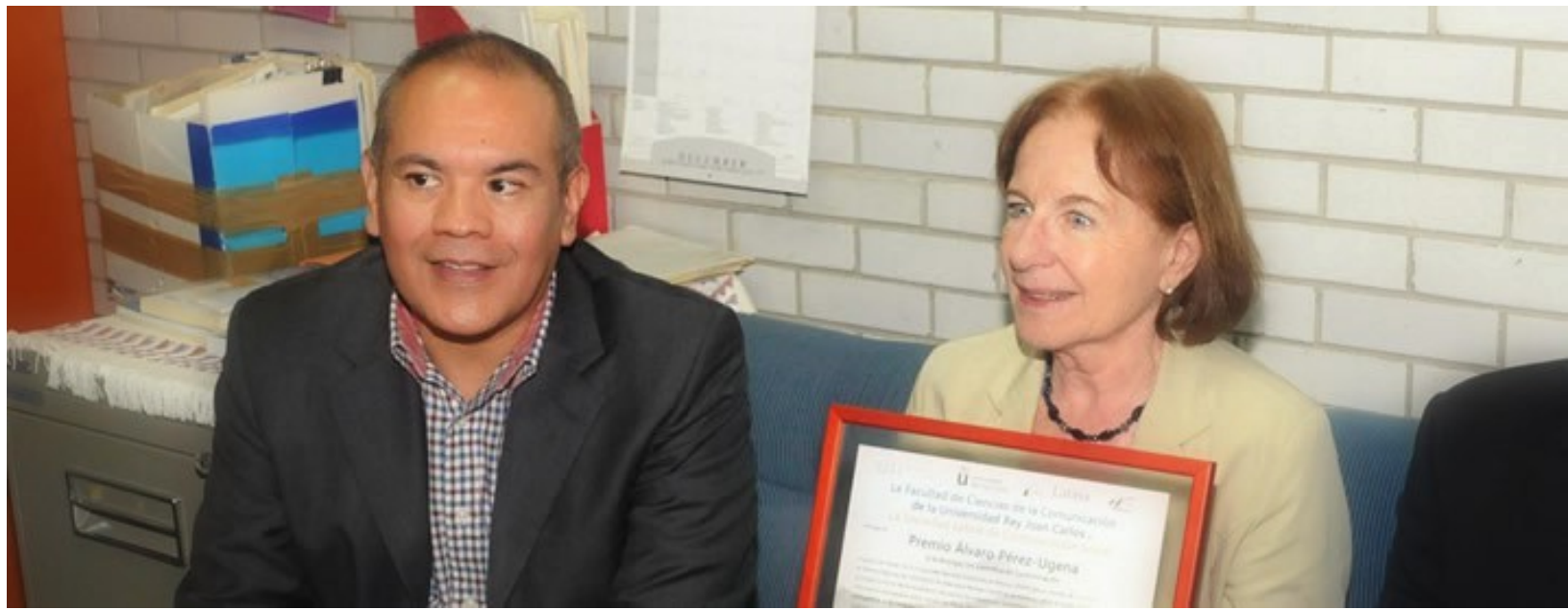

\title{
Profesionalización de la labor editorial de las revistas científicas en América Latina
}

INTER VIEW

\section{Resumen}

Entrevista a Ana María Cetto y José Octavio Alonso Gamboa, coordinadores de Latindex: Sistema Regional de Información en línea para Revistas Científicas de América Latina, el Caribe, España y Portugal.

Palabras clave

Revistas científicas ; Edición científica ; Latindex ; América Latina

\section{Professionalization of the editorial work of journals in Latin America}

\section{Abstract}

Interview with Ana María Cetto and José Octavio Alonso Gamboa, Latindex coordinators: Regional Cooperative Online Information System for Scholarly Journals from Latin America, the Caribbean, Spain, and Portugal.

Keywords

Scholarly Journals ; Scholarly publishing ; Latindex ; Latin America

Es la segunda vez que el equipo de Biblios tiene la oportunidad de reunirse con el equipo coordinador de Latindex, una de las redes con mayor trayectoria y desarrollo en la labor de difusión, promoción y mejora de la accesibilidad y la calidad de las revistas académicas editadas en la región.

Tras cumplir 15 años de actividad ininterrumpida, conversamos con Ana María Cetto y José Alonso sobre los planes futuros de la red además de otras inquietudes entorno al producto de sus logros actuales. 


\section{¿A su criterio, cuales son los mayores logros de Latindex en estos 15 años de actividad?}

Alonso: Pienso que entre los mayores logros de Latindex está la creación del Directorio de Revistas. Este enfoque exhaustivo e inclusivo que tiene Latindex para con las revistas que se editan en América Latina, el Caribe, España y Portugal. Latindex recoge toda la información de las revistas de naturaleza académica, es decir, aquellas que se editan principalmente en las universidades y en las instituciones de educación superior y también de las editadas por las sociedades profesionales.

Creo que uno de los grandes logros del Directorio es que Latindex muestra la industria o la actividad editorial de América Latina, tal como es. Ahí en el Directorio conviven las revistas que han obtenido gran reconocimiento, que tienen circulación internacional, que son reconocidas por sus pares, al igual que otras publicaciones más pequeñas, quizás de interés más local o institucional. Pero todas ellas reflejan de un modo la actividad editorial de América Latina.

Cetto: Hay que considerar que Latindex fue creado hace quince años, yo creo que cuando la mayoría de las revistas de la región no eran consideradas en absoluto, y estaban, yo diría, en peligro de desaparecer. Por lo tanto, el que hoy en día, no solamente que hayan sobrevivido estas revistas, sino que tengan también un espacio, que se les haya dado visibilidad, que se les de a conocer, se me hace que es un logro. O sea, ahora tenemos más de veinte mil títulos de revistas, vivas, activas, y de alguna u otra manera reconocidas por el medio.

Otro logro que me parece muy importante, es que se ha creado una comunidad, trabajamos en red, las instituciones cada una en un país. Y esta red es tremendamente estable, autosustentable, no depende de financiamiento externo, y lo que esto demuestra es que en América Latina, iniciativas como esta pueden sostenerse por quince años y pueden tener mucho futuro por delante.

Alonso: A mí también me gustaría comentar como un logro, la aportación que ha hecho el Catálogo Latindex a las revistas y particularmente a los editores. Cuando no existía el Catálogo Latindex prácticamente el único referente de criterios de calidad editorial eran los internacionales, aquellos sobre todo que utilizaba el antiguo Instituto de Información Científica de Filadelfia, y que como todos sabemos, es bastante sesgado a la publicación internacional, la escrita en inglés, la editada en países desarrollados.

Latindex ha aportado a través de las características editoriales del Catálogo, una plataforma de buenas prácticas editoriales, las mismas que de algún modo no solo ha documentado y ha hecho públicas a través de su sitio web; sino que también ha aprobado por las revistas mismas, y ha compartido con los editores a través de los talleres.

Esta posibilidad de compartir las características de calidad editorial en los talleres con los editores, ha permitido que Latindex incida en este aspecto que Ana María Cetto mencionaba, que es la profesionalización de la actividad editorial en nuestros países.

\section{¿Qué estrategias desarrolla Latindex para fomentar la profesionalización de los editores de revistas científicas en nuestra región?}

Cetto: Una de las debilidades del mundo de la edición de revistas en nuestros países ha sido la falta de profesionalización. Normalmente los editores de las revistas académicas son personas que de buena voluntad han accedido a encargarse de la dirección o de la edición de una revista, sin tener realmente lo antecedentes para ello, ni la preparación adecuada, ni tampoco los recursos adecuados. Entonces normalmente es un trabajo mal remunerado o no remunerado del todo, muy poco reconocido y no profesionalizado. Esto redunda naturalmente en la calidad, la baja calidad del producto, a pesar que hay un esfuerzo personal muy importante.

Entonces pensamos que como parte de este proceso de profesionalización que es necesario que se reconozca que ahí hay una labor que hacer, que requiere de una cierta preparación, que requiere de una cierta capacitación y de una serie de herramientas. 
A través de los talleres para los editores, nosotros ayudamos, y ha habido talleres prácticamente en todos los países, o por región. Pero esta es una labor constante. También damos a los editores asesoría, a través del correo electrónico, del Internet, del Twitter, utilizamos todas las herramientas al alcance hoy en día, pero no nos damos abasto cuando estamos hablando de miles de editores. Entonces también editamos manuales de buenas prácticas, u orientamos a los editores hacia ellos.

Pero también es una cuestión de política y las políticas no las dicta Latindex, las dictan los órganos en nuestros respectivos países, y por ello también abogamos porque se reconozca también la necesidad de profesionalizar esta tarea. $\mathrm{Y}$ creo que hemos avanzado, hay más reconocimiento de esta necesidad, pero creo que hay que continuar desarrollando.

Alonso: El fruto de estas actividades, de los talleres que hablaba Ana María, está reflejado en el sitio web de Latindex. Hay dos pestañas que ofrecen documentación a los editores. Por un lado la pestaña de Documentos, tiene prácticamente todas las presentaciones que hemos dado en los talleres regionales y nacionales que Latindex ha organizado en los últimos 10 años. Allí están todos los materiales para los editores que no se han visto beneficiados con el intercambio directo, puedan hacer consultas a estos materiales.

Y también hay una pestaña en el sitio web denominada Biblioteca del Editor, que va ha ser actualizada y enriquecida con más materiales. Pero ahora la Biblioteca del Editor tiene materiales que son del interés de los editores, que son por ejemplo libros que hablan sobre buenas prácticas editoriales, o bien sobre la publicación electrónica científica actual, el movimiento de acceso abierto, los servicios de indizado y resúmenes, como postular una revista a estos servicios, cuales son los criterios que más prevalecen sobre los servicios de indizado y resumen para incorporar una publicación. En fin, temas que creemos que son de interés y ayuda para todos los editores.

\section{¿Consideran significativo el aporte de Brasil a las iniciativas de editoración científica en la región?}

Cetto: Hoy en día estamos mirando al Brasil como un modelo en muchos sentidos, y creo que también en el mundo de la edición de las revistas se puede aprender mucho de ellos. Esto no es nuevo, Brasil ha sido pionero en varios sentidos, por ejemplo desde hace años tienen una asociación muy fuerte de editores científicos, la ABEC, que ha jugado un papel muy importante. Recuerdo que cuando nosotros empezamos a trabajar en Latindex, la ABEC ya existía, y prácticamente ningún otro país tiene una asociación también organizada. Claro, uno puede decir que país es un país muy grande, bueno pero hay cosas que serían más fáciles de hacer en países más pequeños, no es un pretexto para que no hagamos la tarea en otros países.

Otro sentido que me parece muy importante en cuanto a los modelos, es el de las políticas. Brasil es un país que adopta políticas nacionales y que las pone en práctica y que les da seguimiento. Y eso es desgraciadamente poco común en nuestros países. En particular en el campo de la edición científica, la ausencia de políticas la sienten muchos los editores, los usuarios, las bibliotecas, etc. Entonces actualmente hay una política de información y de acceso a la información bastante clara en Brasil, que yo creo que bien puede ser usada como modelo, no para ser copiada pero como modelo de inspiración y de aliento para los países.

En cuanto a Latindex mismo, creo que la participación de Brasil, no solo como centro de acopio sino como socio de Latindex, nos ha dado una serie de experiencias que se comparten con otras experiencias de manera interesante. Yo pienso que el hecho que el IBICT sea el centro nacional Latindex, es un punto a favor, porque en otros países no se cuenta con un centro nacional de información de la envergadura del IBICT, y eso ha sido una fuente de experiencias y de lecciones para el sistema de Latindex. 
Alonso: Creo que las instituciones asociadas a Latindex, han volteado a ver a Brasil en los últimos años, por la tremenda actividad en la creación y desarrollo de recursos de información dirigidos a las revistas científicas, en particular a la revista científica electrónica, al movimiento de acceso abierto y a la creación de portales para las revistas nacionales.

Creo que esta acción de Brasil que ha hecho de manera muy decidida para aumentar no solo la visibilidad de sus revistas, sino el acceso y uso de las mismas, puede dar muchas enseñanzas a I resto de países de la región.

\section{¿Qué comentarios les merece las críticas de algunos investigadores respecto a que Latindex está haciendo un daño a las revistas de la región, creando un sistema paralelo a los grandes índices internacionales de publicaciones científicas?}

Cetto: Bueno, yo no soy particularmente amiga de modelos, lo que Latindex ha hecho es crear un sistema, que hacía falta, y la necesidad de un sistema de información sobre las revistas era bastante evidente a principios de los noventa y Latindex vino a cubrir un hueco. Primero había que hacer acopio de toda la información sobre las revistas que se editan o el máximo de información, porque no se puede decir que se cuente con el cien por ciento, precisamente para conocer mejor ese universo. Porque ese universo definitivamente no está bien representado, está mal representado en los sistemas internacionales, sobre todo en aquellos sistemas que son selectivos a la hora de indexar y de incorporar títulos.

Ese era nuestro primer objetivo, pero desde un principio nos habíamos planteado un segundo objetivo que era que desarrollemos nuestros propios criterios para ponernos de acuerdo en lo que significa una revista de calidad. No quiere decir que estemos en contra de otros criterios, porque hay que entender que en cada criterio de calidad, el indicador tiene un objetivo detrás. Entonces no se trata de competir con otros sistemas que tienen sus propios indicadores de calidad basados en sus propios criterios, sino que se trata de ver que es lo más representativo, que es lo más útil para elevar la calidad, para concursar, para dar mayor visibilidad a nuestros productos.

Así que yo no siento ninguna competencia. Claro van a seguir habiendo críticas, pero una vez que se acerca a la persona que tiene esas críticas al Sistema de Latindex, con la voluntad de entender que es lo que estamos haciendo y porque lo estamos haciendo, yo creo que esas críticas se desvanecen.

Alonso: Yo creo que esas críticas se han derivado del hecho que no acaban de entender bien, o no quieren entender bien el objetivo de Latindex. El objetivo de Latindex está bien planteado y es público en sus sitio web. Particularmente podemos decir que las características de calidad editorial de Latindex, de ninguna manera son prescriptivas, no son una camisa de fuerza, son como lo hemos dicho, una serie de recomendaciones básicas de buenas prácticas editoriales, basadas sobre todo en la realidad latinoamericana. Esta realidad reflejada en el Directorio que tiene revistas de todo tipo y editores detrás de ellas que vienen de muy diversas experiencias.

Las muy buenas revistas iberoamericanas, y las hay, aquellas que cumplen a cabalidad los requerimientos que dictan los servicios internacionales, pues aquellas están ahí por mérito propio, también están en Latindex y enseñan mucho al resto de las revistas. Pero las demás tienen en las características editoriales de Latindex una plataforma básica, justo para avanzar en lo que estamos hablando, que es profesionalizar la labor editorial, poder hacer mejores revistas y comunicar mejor la ciencia a todos sus niveles: la ciencia que se comunica a través de las revistas de investigación científica, pero también aquella que se comunica a través de las revistas técnico profesionales y las de divulgación, que son muy importantes. Y nuestras revistas también son muy importantes, porque son un vehículo natural de difusión del conocimiento local y regional.

Si este conocimiento local y regional a menudo no es apreciado por los grandes repertorios internacionales, si debe ser apreciado y sin duda, por los usuarios y lectores en la región. 
Cetto: Hay otro aspecto en donde tampoco sabemos si es que algunos colegas no entienden o no quieren entender, y es la diferencia entre lo que es la calidad de una revista, la calidad intrínseca, y por otro lado un prestigio aparentemente asociado al hecho de que esta revista es de acceso reservado, que no es de acceso abierto, esta confusión se maneja bastante en el medio internacional, en muchos círculos, y hay que tener mucho cuidado. Porque hay revistas de acceso abierto, y afortunadamente cada vez más, que son de excelente calidad, que compiten dentro de la calidad intrínseca de sus contenidos, pero con la gran diferencia de que sus contenidos son accesibles a toda la comunidad de usuarios.

Entonces que bueno que se esté dando este movimiento de acceso abierto, y cada vez más el mundo entero tenga precisamente eso: acceso a la bibliografía científica, que de por si, en la mayoría de los casos, ha sido producto de una inversión fuerte por parte de nuestros países, de nuestros estados; y que no tiene porque privatizarse de la manera como ha sucedido en el pasado.

Yo creo que aquí va a haber un cambio, y creo que con este cambio de táctica, y cambio de modelo económico, un cambio de paradigma, y se reconozca el valor de la literatura científica que aparece en acceso abierto.

\section{¿Qué otros aspectos consideran que han sido y son favorables en el desarrollo de Latindex en estos 15 años?}

Alonso: La puerta de entrada de todos los usuarios, editores, bibliotecarios, profesionales de la información, hacia Latindex es su página web. Evidentemente una de las mayores preocupaciones que tenemos es el mejorarla. Tenemos en mente hacer una reingeniería de la página en los próximos 14 meses, que permita más servicios a los usuarios.

Hemos notado que la vasta colección de datos que Latindex ha recogido en los últimos 15 años, sobre todo este amplio universo de revistas académicas que se editan en la región, es de mucho interés para ser explotados. Entonces buena parte de los servicios van a estar enfocados a que los usuarios puedan obtener esos datos, de una manera más sencilla, más práctica, y que de alguna manera los pueda manipular para sus intereses particulares. Y también enriquecer la página web con la múltiple gama de gráficos sobre las revistas científicas que puede ofrecer Latindex. A nivel estadístico podemos ofrecer datos sobre la tendencia de crecimiento de las revistas, sobre todo las revistas electrónicas en los últimos años. La clasificación temática de estas publicaciones, su periodicidad, los países donde se editan. Inclusive por la recogida de tipo histórico que tiene Latindex, es muy útil para ver un poco la pervivencia o la esperanza de vida de las publicaciones en los distintos países.

En Latindex hay países que han encontrado solo en nuestro sitio web, datos interesantes de sus publicaciones, particularmente los países de menor desarrollo, como los países de América Central que tienen ya una gran cantidad importante de información metida en Latindex, y cuyos datos pueden ser de utilidad para fijar políticas alrededor de sus propias revistas académicas.

Cetto: Creo que otro aspecto importante que da un buen futuro a Latindex, un futuro de mucho compromiso, es el estar relacionado con el Encuentro de Redes Universitarias y Consejos de Rectores de América Latina y el Caribe, y que fue convocado por cuatro rectores de universidades latinoamericanas. Este fue un encuentro sobre el ranking de las universidades, sus impactos y sus consecuencias. Y después de varios días de debates muy interesantes y muy intensos, los rectores participantes firmaron una declaración, que también ha sido suscrita por el Sistema Latindex, en el que se hace ver que en la región se necesita trabajar en un mejor conocimiento de la realidad de sus instituciones de educación superior, y esto implica, entre otras cosas, la posibilidad de generar indicadores con criterios propios que reflejen esa realidad, puesto que los sistemas de ranking internacional no la reflejan, o la reflejan de manera muy parcial y pobre.

En este sentido las revistas naturalmente juegan un papel muy importante, las revistas académicas, las revistas científicas; y tener y poder contar con una fuente de información acerca e estas revistas, y que no solamente es una buena fuente de información, sino que es un sistema que está en contacto con las revistas mismas, y que puede dar más información de calidad, que permite conocer este mundo y actuar sobre el. Creo que esto puede ser importante 
cuando los rectores que han firmado esta declaración, pongan manos a la obra y avancen en la definición de esas tareas que se proponen hacer por el mejor conocimiento de la realidad, y para la consecuente posibilidad de evaluar el desempeño que realizan las universidades en nuestro propio contexto, el contexto latinoamericano y el contexto de cada uno de los países.

\section{Producción}

Transcripción y edición: Julio Santillán-Aldana

\section{Agradecimientos}

Sistema Regional de Información en línea para Revistas Científicas de América Latina, el Caribe, España y Portugal (Latindex).

Instituto Brasileiro de Informação em Ciência e Tecnologia (IBICT).

Universidade de Brasília. Faculdade de Ciência da Informação(UnB, FCl).

(c) EY New articles in this journal are licensed under a Creative Commons Attribution 3.0 United States License.

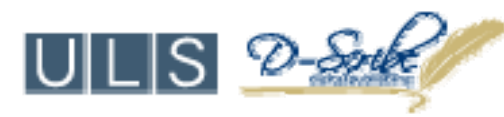

This journal is published by the University Library System of the University of Pittsburgh as part of its $\underline{D-S c r i b e}$ Digital Publishing Program and is cosponsored by the University of Pittsburgh Press. 